\title{
IMPACTO DA COVID-19 EM IDOSOS INSTITUCIONALIZADOS EM ESTRUTURAS RESIDENCIAIS PARA PESSOAS IDOSAS
}

\author{
Maria Helena Pimentel \\ Unidade de Investigação em Ciências da Saúde: Enfermagem (UICISA:E), Instituto Politecnico de Braganca, \\ Campus de Santa Apolonia, 5300-253 Braganca, Portugal. \\ hpimentel@ipb.pt \\ Fernando Pereira \\ Unidade de Investigação e Intervenção educativas da FPCEUP - CIIE, Instituto Politecnico de Braganca, \\ Campus de Santa Apolonia, 5300-253 Braganca, Portugal. \\ Cristina Teixeira \\ Unidade de Investigação EPIUnit - Instituto de Saúde Pública, Universidade do Porto, Instituto Politecnico de \\ Braganca, Campus de Santa Apolonia, 5300-253 Braganca, Portugal.
}

Recepción Artículo: 19 mayo 2021 Admisión Evaluación: 19 mayo 2021 Informe Evaluador 1: 21 mayo 2021 Informe Evaluador 2: 27 mayo 2021 Aprobación Publicación: 01 junio 2021

\section{RESUMO}

A institucionalização dos idosos em Estruturas Residenciais para Pessoas Idosas (ERPI), necessária devido às dinâmicas populacionais e familiares das sociedades ocidentais envelhecidas, sempre levantou questões sobre a qualidade de vida e segurança dos idosos. Vários modelos de institucionalização de longa duração foram teorizados, conceptualizados e levados à prática. Contudo, foi precisamente nestas instituições que 0 efeito da pandemia Covide-19 foi mais devastador. Este estudo tem como objetivo apresentar a evidencia epidemiológica e científica sobre 0 impacto da COVID-19 nos idosos institucionalizados, e perspetivar as mudanças necessárias a introduzir nos modelos de institucionalização.

Procedemos a uma revisão da literatura sobre 0 tema, na base de dados Web of Knowledge utilizando as palavras-chave: "COVID-19" e "Long Term Care Facility", considerando as publicações desde 0 início da pandemia até ao momento da revisão. Foram obtidas 176 publicações. Os critérios de inclusão foram artigos sobre: (1) incidência e mortalidade por infeção COVID-19 em idosos institucionalizados (2) caraterísticas das estruturas residenciais potenciadoras de risco (3) fatores arquitetónicos e organizacionais mitigadoras do impacto da pandemia COVID-19. Foram selecionados 23 cujo texto integral foi analisado: 12 são estudos empíricos; 8 são Relatórios e 3 são Normas Orientadoras.

0 grave impacto da pandemia COVID-19 é explícito nos números reportados. Os fatores que aumentam 0 risco de casos COVID-19 e agravam a dimensão dos surtos nas ERPIs são: (1) relacionados com as estruturas residenciais; (2) relacionados com o estado de saúde dos idosos; (3) gestão de recursos humanos e materiais; 


\section{IMPACTO DA COVID-19 EM IDOSOS INSTITUCIONALIZADOS EM ESTRUTURAS RESIDENCIAIS PARA PESSOAS IDOSAS}

(4) capacitação dos profissionais de saúde; (5) demografia e recursos de saúde; (6) risco social. As estratégias para mitigar o impacto dos surtos são apontadas em linha com os fatores de risco.

As instituiçõ̃es de idosos do tipo Long-Term Care, como as ERPI, revelaram a sua inequívoca exposição ao efeito da pandemia COVID-19, pelo que urge repensar este modelo institucional.

Palavras-chave: COVID-19; idosos; institucionalização; ERPI

\section{ABSTRACT}

Impact of COVID-19 on elderly living long term residences. The institutionalization of the elderly in Long-Term Care Facilities (LTCF), due to the population and family changes in aging western societies, has always raised questions about the quality of life and safety of the elderly. Several LTCF models have been theorized, conceptualized and put into practice. However, it was precisely in these institutions where the COVID-19 pandemic has the most devastating impact. This study aims to present the epidemiological and scientific evidence about the impact of COVID-19 on institutionalized elderly, and to envisage the necessary changes that should be introduced in the LTFC models.

We conducted a literature review in the Web of Knowledge database using the keywords: "COVID-19" and "Long Term Care Facility", considering the publications from the beginning of the pandemic until the moment of the review. 176 citations were obtained. We included articles on: (1) incidence and mortality due to COVID-19 infection in institutionalized elderly (2) characteristics of residential structures which increase the risk of COVID19 infection (3) architectural and organizational factors that mitigate the impact of the COVID-19 pandemic. Thus 23 were selected whose full text was analyzed: 12 are empirical studies; 8 are Reports and 3 are Guidelines.

The serious impact of the COVID-19 pandemic is explicit in the reported figures. The factors that increase the risk of COVID-19 cases and worsens the outbreaks in ERPIs are: (1) related to residential structures; (2) related to the health status of the elderly; (3) human and material resources management; (4) training of health professionals; (5) demographics and health resources; (6) social risk. Strategies to mitigate the impact of outbreaks are identified in line with risk factors.

Long-Term Care elderly institutions, such as ERPIs, have revealed their unequivocal exposure to the effect of the COVID-19 pandemic, so there is an urgent need to rethink this institutional model.

Keywords: COVID-19; elderly; institutionalization; residential structure for elderly; long term care facility

\section{INTRODUÇÃO}

Fragilidades e exposição ao risco em saúde e risco social da atual geração de idosos institucionalizados.

A Estrutura Residencial para Pessoas Idosas (ERPI), o Centro de Dia e o Serviço de Apoio Domiciliário para Idosos (SAD) são as respostas sociais mais representativas dirigidas às Pessoas Idosas. Tem como finalidade assegurar as suas atividades básicas e instrumentais de vida diária, além de visarem a promoção, a inclusão e a participação na comunidade, independentemente do maior ou menor grau de autonomia/dependência do idoso e de este se encontrar a residir na sua habitação ou numa instituição. Em Portugal, em 2018, existiam cerca de 7300 equipamentos sociais destes três tipos (Ministério do Trabalho e da Solidariedade Social, 2019).

Tem sido reconhecido que as Estruturas Residenciais para Pessoas Idosas (ERPI) são locais vulneráveis para a propagação de infeções respiratórias virais. A vulnerabilidade está associada não só ao historial clínico dos idosos residentes e às suas fragilidades e dependência na execução de atividades mas também às caraterísticas das estruturas residenciais que os acolhem (Lansbury, Brown, \& Nguyen-Van-Tam, 2017). Esta vulnerabilidade tornou-se evidente desde que teve início a pandemia por COVID-19 com impacto extremamente nefasto, quer pelo elevado número de infeções e de mortes, observadas nos residentes destas instituições.

Em Portugal as dificuldades das ERPIs são bem conhecidas lotação completa, por vezes até com mais idosos do que a sua capacidade máxima, escassez de pessoal técnico especializado, idosos apresentando muitas fragilidades e dependência. Noutro plano, não raras vezes, estas instituições subsistem com dificuldades financeiras 
e, frequentemente, com modelos de gestão pouco eficazes. Estes constrangimentos tornaram-se particularmente evidentes no atual contexto de pandemia COVID-19, mostrando que os idosos institucionalizados enfrentam dificuldades maiores para conseguirem um envelhecimento com qualidade e segurança. Isto deve-se à conjugação de diversos fatores que potenciam mutuamente num ciclo conducente à perda de qualidade dos serviços disponibilizados.

0 primeiro fator reside no envelhecimento da população portuguesa, $21,7 \%$ de idosos correspondendo a cerca de 2 milhões e 200 mil idosos (PORDATA, 2019). 0 envelhecimento é ainda mais acentuado em municípios do interior do país onde a proporção de idosos ultrapassa um terço da população (Instituto Nacional de Estatística, 2020). Uma verdadeira questão social na medida em que há fortes constrangimentos logísticos, financeiros e de recursos humanos no apoio a tão grande número de idosos para cumprir os critérios de qualidade com base no modelo Person Centered Care (Falcão, Pereira, Pimentel, Teixeira, \& Rodrigues, 2017).

0 segundo fator consiste na institucionalização massiva de idosos. Em 2018, existiam em Portugal 2700 ERPIs com capacidade para acolher 100000 idosos, correspondendo a cerca de 4,5\% do total de idosos (Ministério do Trabalho e da Solidariedade Social, 2019). Esta proporção de idosos institucionalizados em ERPI por oposição aos residentes na comunidade, parece relativamente modesta, todavia ela tem aumentado de forma consistente na última década, fruto das dinâmicas populacionais e familiares registadas na sociedade portuguesa. A população residente nas ERPIs caracteriza-se por níveis elevados de dependência nas atividades básicas e instrumentais de vida diária, muito envelhecida pois mais de 50\% tem idade superior a 80 anos de idade (Ministério do Trabalho e da Solidariedade Social, 2019) com comorbilidades e, frequentemente, polimedicados (Pinto, Pereira, \& Mateos-Campos, 2015). 0 grande número de utentes em ERPIs facilita a transmissão de infeções respiratórias, incluindo a infeção por COVID-19 e a sua fragilidade aumenta o risco de ocorrência das formas mais graves de doença (Lansbury et al., 2017).

0 terceiro fator releva que a maioria das ERPIs tem edifícios únicos e/ou de grandes dimensões nos quais as atividades diárias, quer as básicas (alimentação por exemplo) quer as instrumentais e ocupacionais, decorrem de forma demasiado próxima e massificada. Acresce que pessoal mais especializado como médicos, enfermeiros, psicólogos, entre outros, frequentemente, prestam serviços em mais do que uma instituição de saúde ou social, funcionando como vetores de transmissão das infeções (Childs et al., 2019; Dosa, Jump, Laplante, \& Gravenstein, 2020; Mas Romero et al., 2020; McMichael et al., 2020).

0 quarto fator emerge do facto de que a maioria das ERPIs terem estatuto de IPSS (Instituição Particular de Solidariedade Social). Isto significa que têm acesso a subvenções estatais indispensáveis mas sempre insuficientes para a otimização dos serviços prestados. Acresce a frequente adoção de modelos de gestão pouco eficientes e uma crónica insuficiência de recursos humanos que pode dificultar 0 apoio destes idosos com segurança face a um surto epidémico.

0 quinto fator consiste na matriz cultural da atual geração dos idosos que valoriza os vínculos familiares e sociais. Mesmo em situações de normalidade os idosos institucionalizados em ERPIs sofrem frequentemente de isolamento social, risco agravado pela necessidade de distanciamento social para contenção da pandemia, acentuando-se 0 sentimento de vazio, de perda de identidade, que pode levar à desesperança, no sentido de Erikson (1972). É verdade que as instituições se esforçam por manter os laços sociais e familiares, fazendo uso, por exemplo, das redes sociais, mas é um facto que as visitas presenciais ou saídas da instituiç̧ão estão muito limitadas ou interditas e o contacto pessoal reduzido ao mínimo.

A pandemia por COVID-19 que assolou o mundo teve impacto particularmente agravado entre idosos institucionalizados. É objetivo deste estudo apresentar a evidencia epidemiológica e científica sobre o impacto da COVID-19 nos idosos institucionalizados, assim como repensar o planeamento e os modelos de gestão das ERPIs no sentido de aumentar a qualidade de vida e a segurança de idosos institucionalizados. 


\section{IMPACTO DA COVID-19 EM IDOSOS INSTITUCIONALIZADOS \\ EM ESTRUTURAS RESIDENCIAIS PARA PESSOAS IDOSAS}

\section{METODOLOGIA}

Para 0 efeito procedemos a uma revisão da literatura sobre o tema, considerando as publicações desde 0 início da pandemia COVID-19. Fez-se a pesquisa de artigos na base de dados Web of Knowledge utilizando as seguintes palavras-chave: "COVID-19" e "long term care facility". Foi considerado o período temporal de publicação.

As publicações obtidas foram escrutinadas de forma a selecionar artigos que apresentem resultados sobre (1) incidência e mortalidade por infeção COVID-19 em idosos institucionalizados (2) caraterísticas inerentes às estruturas residenciais que potenciam o risco de infeção (3) fatores arquitetónicos e organizacionais que diminuem 0 impacto da pandemia COVID-19 em idosos institucionalizados.

Foram obtidas 176 publicações e após escrutínio dos títulos e resumos foram selecionadas 23 cujo texto integral foi analisado. Destas 12 são estudos empíricos (Abrams, Loomer, Gandhi, \& Grabowski, 2020; Barnett, Hu, Martin, \& Grabowski, 2020; Blain et al., 2020; Brown et al., 2020; Fisman, Bogoch, Lapointe-Shaw, McCready, \& Tuite, 2020; Gandal, Yonas, Feldman, Pauzner, \& Tabbach, 2020; He, Li, \& Fang, 2020; Mas Romero et al., 2020; Montoya et al. 2020; 0'Caoimh et al., 2020; Rolland et al., 2020); 8 são Relatórios (Harris et al., 2020; Kim, 2020; Kuzuya et al., 2020; Lansbury et al., 2017; Lester, Holahan, Siskind, \& Healy, 2020; McMichael et al., 2020; Mills et al., 2020; Mitchell et al., 2020) e 3 são Normas Orientadoras (Danis et al., 2020; Dosa et al., 2020; Yen et al., 2020).

\section{RESULTADOS}

Apresentam-se os resultados da pesquisa em duas tabelas. A Tabela 1 estruturou-se nos seguintes fatores de análise: autores do estudo e país; tipo de estudo; período temporal a que se reportam os dados, a maioria corresponde aos primeiros meses da pandemia, primeiro surto pandémico, no ano 2020; tipo de ERPI; dimensão e impacto da COVID-19 nas Estruturas Residenciais Para Idosos (ERPIs).

0 grave impacto da pandemia COVID-19 nas ERPIs está explícito nos números reportados nas duas tabelas apresentadas. Cerca de $12 \%$ do total de infetados reportados nos EUA correspondem à fatia da população institucionalizada em ERPIs (He et al., 2020). Só nos primeiros três meses desde o início da pandemia, 30 a $70 \%$ das ERPIs nos EUA (Abrams et al., 2020; He et al., 2020) e 43\% das ERPIs no Canadá (Fisman et al. 2020) enfrentaram situações de infeção COVID-19. A taxa de infetados entre residentes das ERPIs, 2 a 4 semanas desde a deteção de um caso positivo variou entre 7\% a 48\% (Blain et al., 2020; Brown et al., 2020; Mas Romero et al., 2020; Montoya et al., 2020). Cerca de 10\% de residentes das ERPIs foram hospitalizados devido à infeção por COVID-19 (Mas Romero et al., 2020) e considerando especificamente residentes infetados por COVID-19 a proporção de hospitalizados atingiu 54,5\% (McMichael et al., 2020). Mais grave foi o impacto da infeção na mortalidade de residentes das ERPIs. Na Europa, durante as primeiras semanas da pandemia a população residente de instituições Long Term Care e infetada por COVID-19 contribuiu para uma proporção de óbitos por COVID-19 que variou entre $31 \%$ a $66 \%$ do total de óbitos por COVID-19 em cada país estudado (Danis et al., 2020). Nos EUA 27\% das mortes por COVID-19 ocorreram em residentes de ERPIs (HE et al., 2020). No Canadá, os idosos residentes em LTCF apresentaram 13 vezes mais risco de morte por COVID-19 em comparação com os idosos da comunidade (Fisman et al., 2020). Foram reportados valores letalidade por COVID-19 entre residentes de ERPIs que variaram entre 13\% e 83\% (Blain et al, 2020; Danis et al., 2020; Mas Romero et al., 2020). 
Tabela 1 - Impacto da COVID-19 em ERPIS

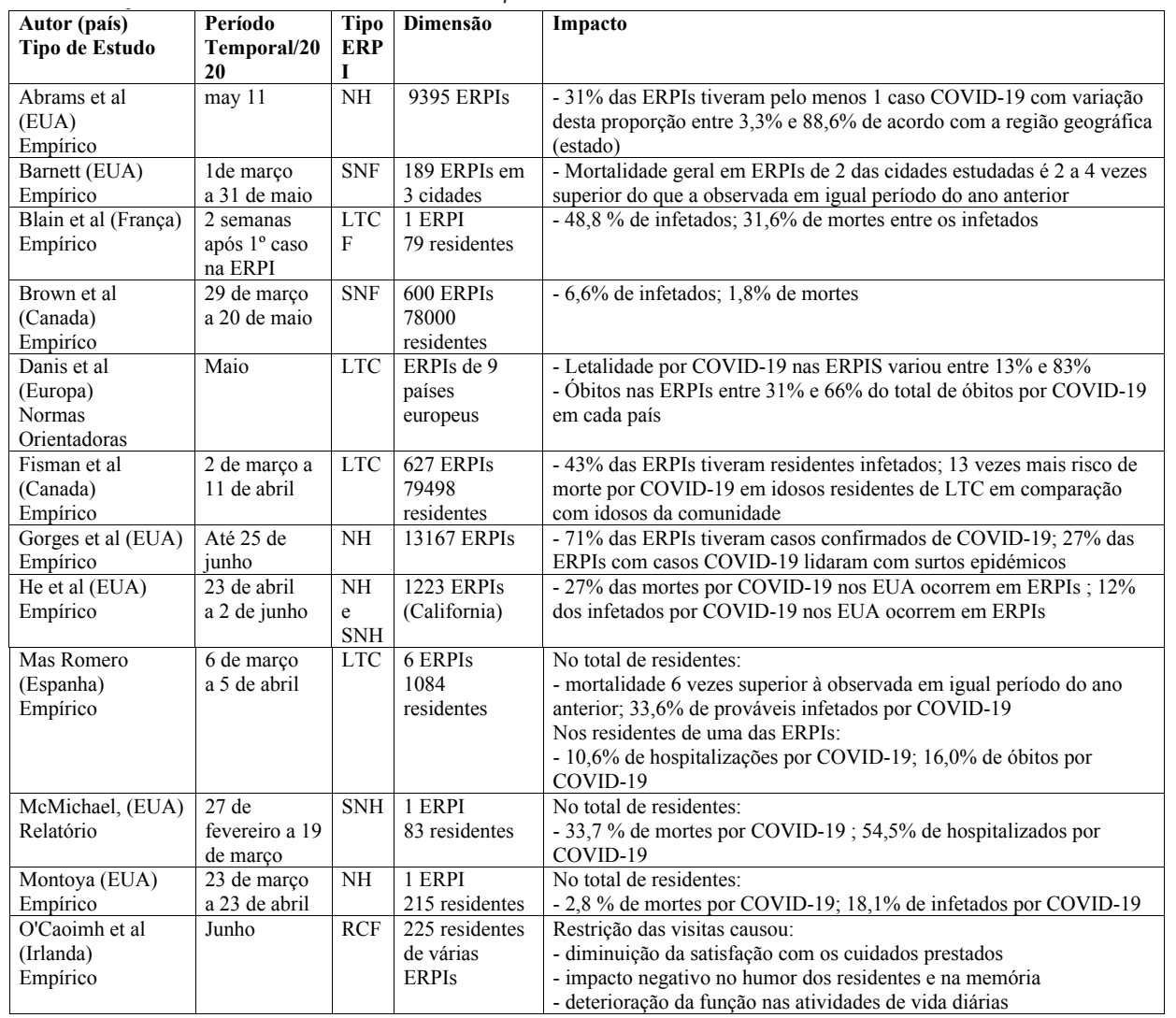

LTCF; Long term care facility; NH; Nursing Home; PALTCF; Post-Acute Long Term Care Facility; RCF; Residential Care Facility; SNF; Skilled nursing facility; SNH; Skilled nursing home

A Tabela 2 reporta os fatores de risco da COVID-19, bem como as estratégias/soluções para mitigar o impacto dos surtos da infeção viral na população idosa institucionalizada.

Aumentam o risco de casos COVID-19 e agravam a dimensão dos surtos nas ERPIs, de acordo com os estudos analisados, um conjunto de fatores (1) relacionados com as estruturas residenciais: elevado número de camas e índice de aglomeração dentro das ERPIs (Abrams et al., 2020; Brown et al., 2020; Gandal et al., 2020: He et al., 2020; Lansbury, 2020); ERPIs com fins lucrativos (Abrams et al., 2020; Brown et al., 2020; He et al., 2020) e localização urbana (Abrams et al., 2020); ERPIs com financiamento público (Rolland et al., 2020); insuficiente dotação orçamental (Kuzuya, 2020); ERPIs de baixa qualidade e edifícios antigos (He et al., 2020); transferência de residentes entre instituiç̧ões (McMichael et al., 2020); espaço insuficiente para isolamento, quarentena e criação de grupos de acordo com o estatuto face à infeção; partilha de quartos com proximidade entre camas (Kim et al., 2020); (2) relacionados com 0 estado de saúde dos idosos: maior fragilidade; idade avançada; comorbilidades crónicas (Abrams et al., 2020; Danis et al., 2020; Harris et al., 2020: Mas Romero et al., 2020; Lansbury, 2020) particularmente doença cardíaca e doença respiratória crónica (Dosa et al., 2020); deterioração da função motora (Mas Romero et al., 2020); 0 risco de morte por COVID-19 é 12 vezes maior na situação de doença cardíaca e 7 vezes maior na situação de doença respiratória crónica (Dosa et al., 2020); (3) gestão de 


\section{IMPACTO DA COVID-19 EM IDOSOS INSTITUCIONALIZADOS EM ESTRUTURAS RESIDENCIAIS PARA PESSOAS IDOSAS}

recursos humanos e materiais: rotação e partilha de cuidados e de profissionais de saúde entre instituições, (Blain et al., 2020; Danis et al., 2020; Kim et al., 2020; Lansbury, 2020, McMichael et al., 2020, Rolland et al., 2020); inadequação do número de horas de trabalho da equipa de enfermagem em função do aumento de casos COVID-19 (Gorges et al., 2020); insuficiente dotação de pessoal; insuficiente investimento em material e medidas de proteção e controlo (Kuzuya, 2020); deteção de infeção em assintomáticos (Lester et al., 2020); receber visitas (0'Caoimh et al., 2020, Lansbury, 2020); (4) capacitação dos profissionais de saúde: falta de consciência para o problema; falta de preparação das equipas profissionais (Kuzuya, 2020); (5) demografia e recursos de saúde: maior densidade populacional na comunidade; menor $n^{0}$ de camas hospitalares per capita na comunidade (Gandal et al., 2020) (6) risco social: o risco agravado de isolamento social enquanto medida para conter a propagação da infeção é descrito por O'Caoimh et al. (2020), através da restrição das visitas, causando diminuição da satisfação com os cuidados prestados; impacto negativo no humor dos residentes e na memória, bem como alteração e deterioração das funcionais atividades de vida diárias.

Já no que diz respeito às estratégias e soluções para mitigar o impacto dos surtos da infeção viral na população idosa institucionalizada são apontadas em linha com os fatores de risco e englobam medidas aplicadas a residentes, equipa e visitantes estabelecidas de acordo com o nível de risco na comunidade como testagem para deteção precoce de infetados sintomáticos e assintomáticos (Abrams et al., 2020; Dosa et al., 2020; Harris et al.,2020; Lansbury, 2020; Montoya, 2020); medidas de higiene (Abrams et al., 2020; Danis et al., 2020; Harris et al., 2020; Lansbury, 2020; Yen et al., 2020) e material de proteção para todos os profissionais (Lester, 2020; Montoya, 2020; Yen et al., 2020); regras de distanciamento; evitar a partilha de profissionais, avaliação de sintomas em visitantes, testagem de visitantes considerados de risco e quando a situação na comunidade é considerada de risco elevado, limitar o número de visitantes (Abrams et al., 2020; Danis et al., 2020; Harris et al., 2020; Mills et al., 2020; Montoya, 2020; Yen et al., 2020); visitas através de janelas (Ickert et al., 2020); manter atividades que permitam distanciamento e videochamadas entre residentes e familiares (Lester, 2020); reduzir 0 número de residentes que partilham o quarto (Blain et al., 2020) e prestar cuidados nos quartos para reduzir contactos (Blain et al., 2020; Montoya, 2020); equipas fixas de profissionais afetos à ERPI e reforço das equipas (Dosa et al., 2020; Mitchell et al., 2020); aumento do número de horas de trabalho das equipas de enfermagem (Gandal et al., 2020); valorização de espaços amplos, múltiplos espaços de lazer e valorização de espaços exteriores (Estabrooks et al., 2020; Ickert et al., 2020); espaços próprios para residentes infetados e preparar as equipas para prestar cuidados a estes residentes (Lester, 2020; Montoya, 2020; Yen et al., 2020); espaço para refeições e descanso dos profissionais (Lester, 2020); criação sistemática de protocolos partilhados em rede entre ERPIs, identificação de necessidades nos residentes e cuidados diferenciados; coordenação dos cuidados nas transferências bidirecionais entre a ERPI e o hospital (Harris et al.,2020); partilha de informação clínica com residentes e seus familiares, comunicação entre profissionais de saúde, considerar a vontade de residentes e familiares relativamente aos cuidados a serem prestados (Kuzuya 2020; Mitchell et al., 2020; Montoya, 2020); formação de profissionais de saúde (Mills et al., 2020; Montoya, 2020; Yen et al., 2020); vacinação; tratamento antiviral e profilaxia (Lansbury, 2020); centros de coordenação para identificação rápida de surtos (Mitchell et al., 2020); identificação precoce e comunicação dos sintomas (Yen et al., 2020), Tele-Health, protocolos partilhados em rede entre ERPIs como medida para diminuir a hospitalização e a mortalidade (Harris et al., 2020); 


\section{Tabela 2 - Fatores de risco da Covid19 e estratégias/soluções para mitigar o impacto dos surtos da infeção} viral na população idosa institucionalizada.

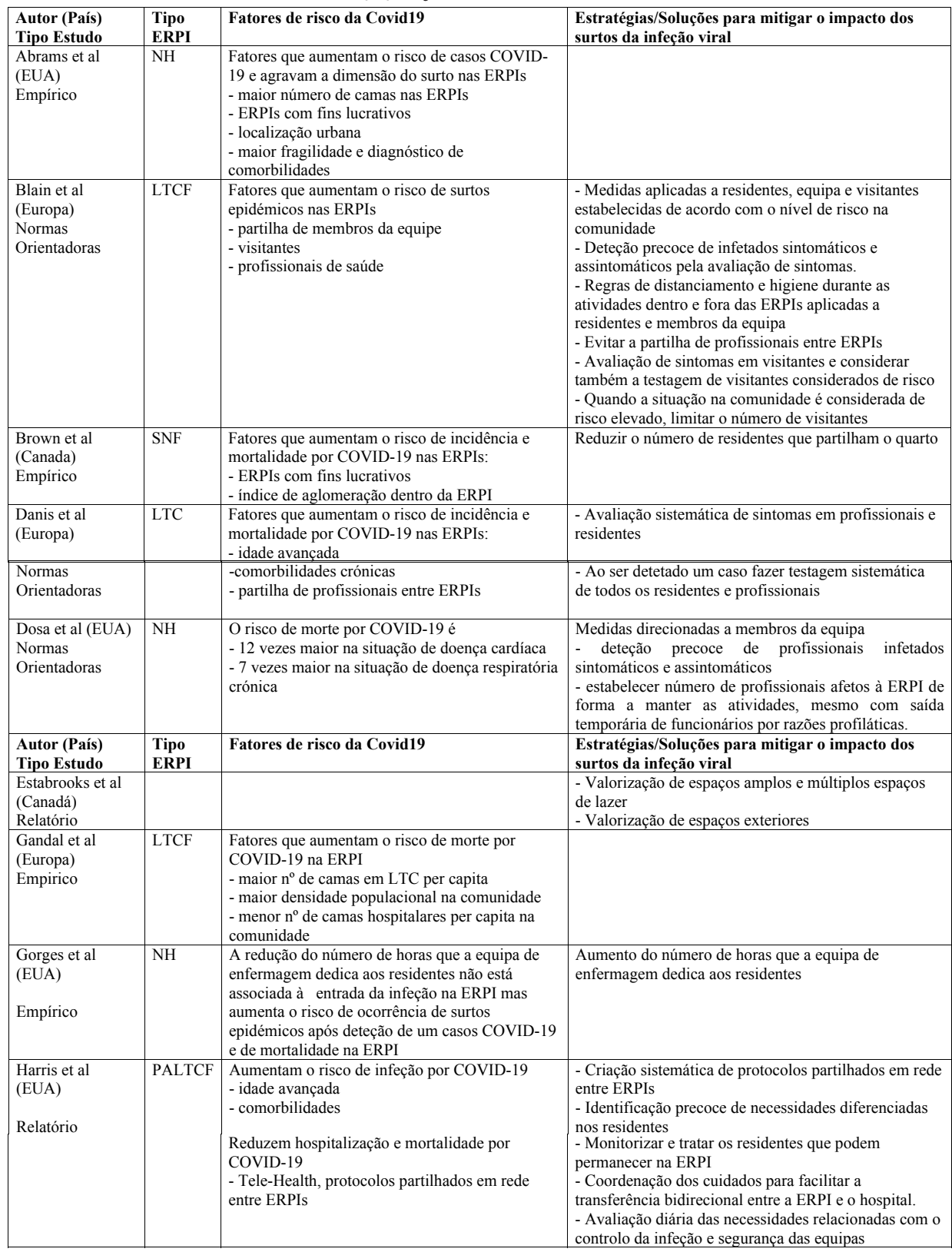




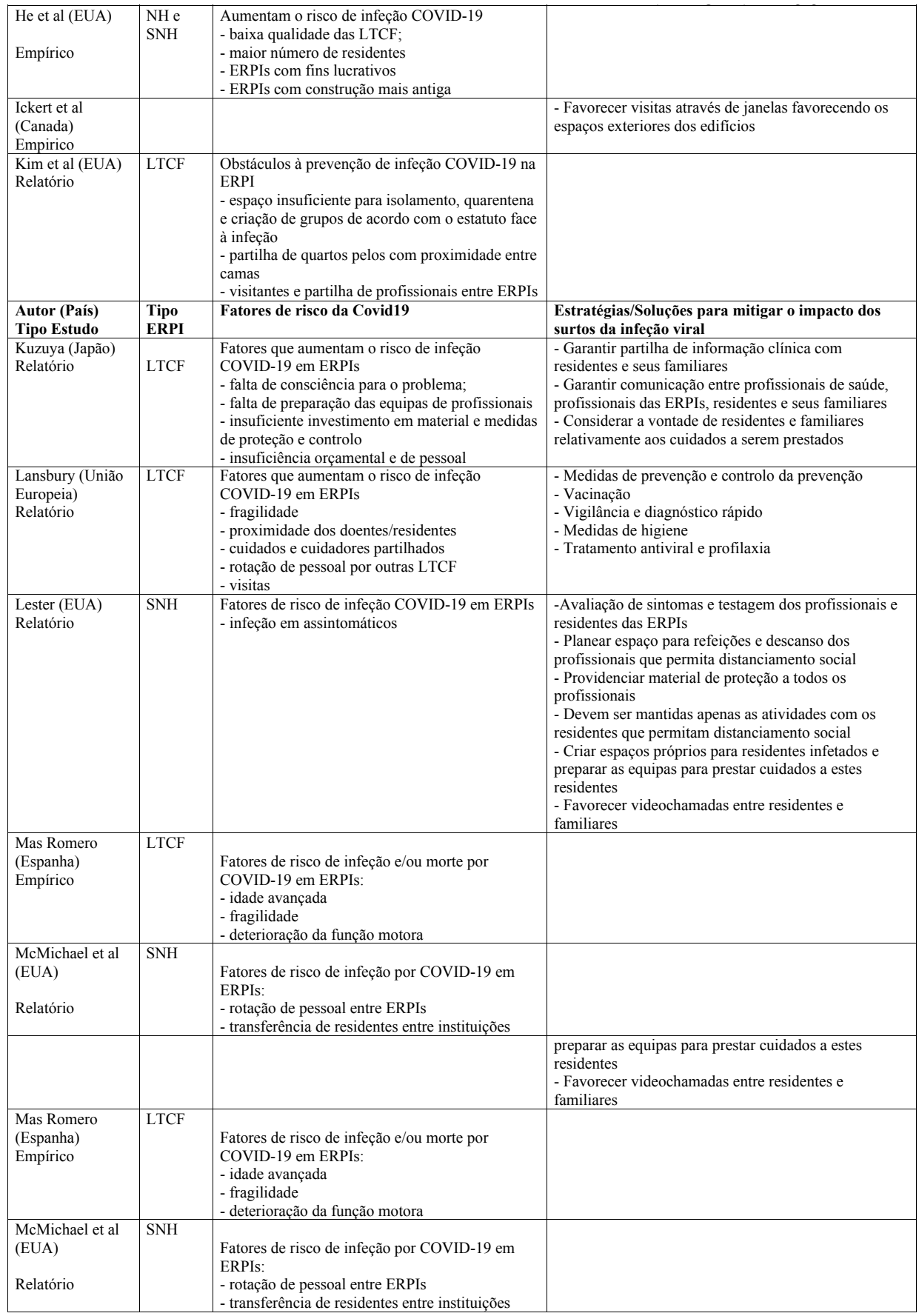




\begin{tabular}{|c|c|c|c|}
\hline $\begin{array}{l}\text { Autor (País) } \\
\text { Tipo Estudo }\end{array}$ & $\begin{array}{l}\text { Tipo } \\
\text { ERPI }\end{array}$ & Fatores de risco da Covid19 & $\begin{array}{l}\text { Estratégias/Soluções para mitigar o impacto dos } \\
\text { surtos da infeção viral }\end{array}$ \\
\hline $\begin{array}{l}\text { Mills et al (EUA) } \\
\text { Relatório }\end{array}$ & ALF & & $\begin{array}{l}\text {-Implementação de medidas de prevenção e controlo de } \\
\text { infeção através da formação de profissionais das ERPIs } \\
\text {-Avaliação de sintomas em profissionais e } \\
\text { implementação de medidas de isolamento para } \\
\text { infetados }\end{array}$ \\
\hline $\begin{array}{l}\text { Mitchell et al } \\
\text { (EUA) } \\
\text { Relatório }\end{array}$ & LTCF & & $\begin{array}{l}\text { - Comunicação rápida e eficaz e partilha de informação } \\
\text { entre as diferentes partes interessadas } \\
\text { - Centros de coordenação permitem identificação } \\
\text { rápida de surtos } \\
\text { - Monitorização da ocupação de ERPIS e da falta de } \\
\text { profissionais para suprir as necessidades. }\end{array}$ \\
\hline $\begin{array}{l}\text { Montoya (EUA) } \\
\text { Empírico }\end{array}$ & $\mathrm{NH}$ & & $\begin{array}{l}\text {-Testagem sistemática } \\
\text {-Isolamento dos positivos } \\
\text { - Manter comunicação entre residentes, profissionais e } \\
\text { famílias; } \\
\text {-Disponibilidade, de equipamento de proteção } \\
\text { individual e formação para utilização correta desse } \\
\text { equipamento } \\
\text { - Residentes permanecem nos seus próprios quartos } \\
\text { onde recebem os cuidados que necessitam } \\
\text { - Estabelecer uma área própria para residentes } \\
\text { infetados }\end{array}$ \\
\hline $\begin{array}{l}\text { Rolland et al } \\
\text { (França) } \\
\text { Empirico }\end{array}$ & LTCF & $\begin{array}{l}\text { Fatores de risco de infeção por COVID-19 em } \\
\text { ERPIs: } \\
\text { - ERPIs com financiamento público } \\
\text { - compartimentar profissionais e residentes em } \\
\text { espaços próprios }\end{array}$ & \\
\hline $\begin{array}{l}\text { Yen et al } \\
\text { (Taiwan) }\end{array}$ & LTCF & & $\begin{array}{l}\text { - Formação e treino de profissionais sobre medidas de } \\
\text { prevenção e controlo da infeção } \\
\text { - Preparar profissionais e os próprios residentes para a } \\
\text { identificação e comunicar sintomas } \\
\text { - Disponibilidade de equipamento de proteção } \\
\text { individual } \\
\text { - Manter o ambiente limpo e desinfetado } \\
\text { - Restringir entrada de indivíduos externos à ERPI ao } \\
\text { estritamente necessário } \\
\text { - Avaliar sintomas e risco em visitantes } \\
\text { - Separação de áreas de acordo com o risco }\end{array}$ \\
\hline
\end{tabular}

\section{DISCUSSÃO}

A percentagem elevada de casos positivos de COVID-19, infeção causada pelo coronavírus 2 da síndrome respiratória aguda grave (SARS-CoV-2), nas unidades de Long Term Care e as elevadas taxas de morbilidade e mortalidade nestas estruturas, como a totalidade dos estudos analisados das tabelas 1 e 2 claramente demonstram, tem posto a nu uma realidade até então oculta o que deve motivar uma reflexão aprofundada sobre este tipo de instituições (Gandal et al., 2020; Gardner, States, \& Bagley, 2020).

Num contexto em que cada vez maior número de idosos carece de apoio para as atividades básicas e instrumentais da vida diária e acabam por ser institucionalizados para garantir esse apoio o cenário observado nas unidades de Long Term Care, em consequência da pandemia COVID-19, levanta questões pertinentes e exige reflexão relativamente ao apoio assistencial aos idosos. Os resultados obtidos realçam a importância de repensar estas unidades valorizando a segurança face a fatores de risco em saúde.

A COVID-19 veio, sem dúvida, reforçar a urgência de repensar o modelo de institucionalização de idosos. A urgência desta situação encontra eco nos indicadores demográficos nacionais (INE, 2020, PORDATA, 2019) e internacionais. Ao invés de um depósito de idosos desvinculados da vida ativa e social delinear um modelo que proporcione privacidade e segurança, manutenção de hábitos e vida, relações sociais e acompanhamento por parte das pessoas mais próximas, uma mudança necessária, mas positiva e gratificante para todos nesta fase mais adiantada da vida. 


\section{IMPACTO DA COVID-19 EM IDOSOS INSTITUCIONALIZADOS EM ESTRUTURAS RESIDENCIAIS PARA PESSOAS IDOSAS}

Urge repensar a institucionalização em função dos fatores de risco da COVID-19 identificados, mitigar 0 impacto dos surtos da infeção viral, garantir a qualidade de vida e segurança dos idosos institucionalizados, face a situações pandémicas.

0 contexto da pandemia COVID-19 e as suas dramáticas consequências nos idosos institucionalizados devem ser a premissa para se repensar a arquitetura e a organização destas estruturas. Tem sido defendido que as ERPIs devem ser organizadas com base em modelos Person Centered Care em que o foco de interesse está no utente, nas suas necessidades e nas suas expetativas (Athwal et al., 2014). Neste modelo, dentro do que é possível face à gradual deterioração das capacidades cognitivas dos idosos, o residente da ERPI participa nas decisões relacionadas com a sua saúde, as suas atividades e 0 seu bem-estar (Williams, Hadjistavropoulos, Ghandehari, Yao, \& Lix, 2015), valorizam-se vários aspetos incluindo a qualidade da estrutura residencial em termos de arquitetura, conforto e adequação do espaço, eficiência da organização. Cuidados adaptados à necessidade dos idosos, permitindo as interações sociais e atividades ocupacionais diversificadas, mas também a comunicação, informação e participação na tomada de decisão (Pereira et al., 2019). A qualidade é avaliada através do nível de satisfação do utente (ou seus familiares), deve ser monitorizada ao longo do tempo de forma a perceber fragilidades que comprometam a satisfação do utente e, por conseguinte, a qualidade da instituição (Falcão, Pereira, Pimentel, Teixeira \& Rodrigues, 2017).

Mantendo estes padrões de qualidade é necessário agora introduzir nas ERPIs aspetos organizacionais e/ou arquitetónicos que aumentem a segurança do idoso face a potenciais riscos para a sua saúde sem comprometer as interações sociais.

Tem sido demonstrado que um elevado número de idosos na instituição é um fator de risco para a ocorrência de surtos de infeções virais, tal como se comprovou neste contexto da pandemia COVID-19 (Abrams et al. 2020, Brown et al., 2020; Gandal et al., 2020; He et al., 2020). Apesar das vantagens organizacionais e financeiras das grandes estruturas onde é possível alocar vários profissionais de saúde de diferentes áreas e proporcionar diversidade de atividades, há o aspeto negativo de concentração de elevado número de utentes, muito heterogéneos relativamente ao nível de dependência e comorbilidades. As estruturas devem ser pensadas para um menor número de utentes (Abrams, Loomer, Gandhi, \& Grabowski, 2020). Áreas comuns amplas e diversificadas são fundamentais para permitir algum grau de interação social com menor risco de transmissão de infeções. 0 espaço individual do utente deve ser também valorizado para que possa usufruir de conforto, evitando longos períodos de tempo em salas comuns partilhadas com outros utentes. Os espaços verdes exteriores são importantes quando se pensa situações de isolamento profilático favorecendo a permanência ou até as atividades ao ar livre (Estabrooks et al., 2020,). Estes espaços podem também ser usados para interação social com familiares e amigos reduzindo o risco de transmissão da doença no interior da unidade (Ickert et al., 2020).

As interações socias não devem ser negligenciadas tendo em conta os efeitos adversos que o sentimento de isolamento e solidão causam no idoso (Robb et al., 2020). 0 risco agravado de isolamento social enquanto medida para conter a propagação da infeção é descrito por O'Caoimh et al., (2020), através da restrição das visitas, causando diminuição da satisfação com os cuidados prestados; impacto negativo no humor dos residentes e na memória, bem como alteração e deterioração das funcionais atividades de vida diárias. Embora as visitas aos utentes possam representar um risco de infeção é necessário salientar a sua importância no bem-estar do idoso. Por conseguinte, os esquemas de visitas devem ser planeados pesando vantagens e riscos em função do bemestar do utente (Estabrooks et al., 2020). Para além de espaços amplos, diversificados, valorizando os espaços exteriores onde as visitas de familiares possam acontecer com menor risco, as ERPIs devem estar preparadas com as novas tecnologias para que os idosos possam estabelecer contacto regular com os seus entes queridos (Ickert et al., 2020; Lester et al, 2020).

0 grande desafio no desenho de unidades Long Term Care são os utentes com elevado grau de dependência, com perda acentuada das capacidades cognitivas ou com diagnóstico de demência (Abrams et al., 2020; Danis et al., 2020). As unidades que prestam cuidados de saúde a utentes que permanecem no leito por longos períodos 
de tempo devem ser planeadas de forma a que as enfermarias sejam amplas e com número mínimo de internamentos 0 que é fundamental para reduzir o risco de infeção (Gandal et al., 2020; Kim et al. 2020; Lansbury et al., 2017, Brown et al., 2020).

Os profissionais que prestam cuidados a estes utentes são peças fundamentais na criação de ambientes confortáveis e seguros. Deve ser providenciada preparação para que estes profissionais possam prestar cuidados com o máximo de qualidade e segurança (Estabrooks et al., 2020). A partilha de profissionais especializados por várias unidades é sem dúvida um risco acrescido no que concerne à transmissão da infeção, pelo que deve ser evitado (McMichael et al., 2020; Blain et al., 2020; Danis et al., 2020). Isto implica investimento em equipas consolidadas, coesas e multidisciplinares em cada ERPI, embora este aspeto possa levantar grandes constrangimentos financeiros.

A monitorização da qualidade das ERPIs é outro aspeto importante assegurando que os utentes recebem os cuidados adequados às suas necessidades. As ERPIs devem ter no seu quadro de pessoal indivíduos responsáveis pela produção e análise de dados estatísticos que permitam monitorizar com transparência os eventos que implicam a segurança e bem-estar dos utentes. Um aspeto que não pode ser ignorado é a disponibilidade para informar familiares e entidades de saúde e/ou segurança social sobre o que se passa na instituição para que os problemas possam ser abordados e resolvidos atempadamente (Kuzuya et al., 2020).

Por fim, é destacada a importância de, pelo menos à escala regional, haver um sistema de networking muito bem articulado, permitindo um planeamento dos cuidados e uma intervenção rápida quando necessário, entre estas instituições de idosos, as unidades de saúde e os centros de produção de conhecimento científico e técnico (Harris et al, 2020; Kuzuya, 2020; Mitchell et al, 2020).

\section{CONCLUSÕES}

As instituições de idosos do tipo Long-Term Care, como as ERPI, revelaram a sua inequívoca exposição ao efeito da pandemia COVID-19. A literatura científica e técnica já fazia referência à vulnerabilidade dos idosos e colaboradores destas instituições, nomeadamente, do risco de contágio das doenças respiratórias. Digamos que o problema estava identificado, mas adormecido, como igualmente refere a literatura, e muito pouco foi sendo feito para o mitigar.

A evolução das ERPIs foi sempre no sentido de proporcionar melhor qualidade de vida e melhores cuidados de saúde aos idosos institucionalizados. 0 próprio modelo Personal Centered Care foi sendo pensado e desenvolvido para garantir esses objetivos, juntamente com a garantia de maior privacidade e respeito pela liberdade dos idosos. 0 efeito de uma pandemia como o COVID-19 não foi de todo antecipado. A segurança dos idosos institucionalizados e dos seus cuidadores escapou no essencial ao escrutínio técnico e científico. Nós próprios, no nosso percurso de cerca de uma década a investigar sobre estas matérias, não antecipámos esta possibilidade.

Esta reflexão é um contributo para um novo paradigma na conceptualização das ERPIs e outras estruturas do tipo Long-Term Care Facilities.

\section{REFERÊNCIAS BIBLIOGRÁFICAS}

Abrams, H. R., Loomer, L., Gandhi, A., \& Grabowski, D. C. (2020). Characteristics of U.S. Nursing Homes with COVID 19 Cases. J Am Geriatr Soc, 68(8), 1653-1656. doi:10.1111/jgs.16661

Athwal, L., Marchuk, B., Laforêt-Fliesser, Y., Castanza, J., Davis, L., \& LaSalle, M. (2014). Adaptation of a best practice guideline to strengthen client-centered care in public health. Public Health Nurs, 31(2), 134-143. doi:10.1111/phn.12059

Barnett, M. L., Hu, L., Martin, T., \& Grabowski, D. C. (2020). Mortality, Admissions, and Patient Census at SNFs in 3 US Cities During the COVID-19 Pandemic. Jama, 324(5), 507. doi:10.1001/jama.2020.11642

Blain, H., Rolland, Y., Schols, J., Cherubini, A., Miot, S., O'Neill, D., .. Benetos, A. August 2020 Interim EuGMS guidance to prepare European Long-Term Care Facilities for COVID-19. European Geriatric Medicine. doi:10.1007/s41999-020-00405-z 
Brown, K. A., Jones, A., Daneman, N., Chan, A. K., Schwartz, K. L., Garber, G. E., . . Stall, N. M. (2020). Association Between Nursing Home Crowding and COVID-19 Infection and Mortality in Ontario, Canada. JAMA Internal Medicine. doi:10.1001/jamainternmed.2020.6466

Childs, A., Zullo, A. R., Joyce, N. R., McConeghy, K. W., Van Aalst, R., Moyo, P., ... Gravenstein, S. (2019). The burden of respiratory infections among older adults in long-term care: a systematic review. BMC Geriatrics, 19(1). doi:10.1186/s12877-019-1236-6

Danis, K., Fonteneau, L., Georges, S., Daniau, C., Bernard-Stoecklin, S., Domegan, L., . . Schneider, E. (2020). High impact of COVID-19 in long-term care facilities, suggestion for monitoring in the EU/EEA, May 2020. Eurosurveillance, 25(22). doi:10.2807/1560-7917.es.2020.25.22.2000956

Direção Geral de saúde (DGS) . Atualização Oficial COVOD-19 em Portugal. Lisboa: DGS. . (2020).

Dosa, D., Jump, R. L. P., Laplante, K., \& Gravenstein, S. (2020). Long-Term Care Facilities and the Coronavirus Epidemic: Practical Guidelines for a Population at Highest Risk. Journal of the American Medical Directors Association, 21(5), 569-571. doi:10.1016/j.jamda.2020.03.004

Fisman, D. N., Bogoch, I., Lapointe-Shaw, L., McCready, J., \& Tuite, A. R. (2020). Risk Factors Associated With Mortality Among Residents With Coronavirus Disease 2019 (COVID-19) in Long-term Care Facilities in Ontario, Canada. Jama Network Open, 3(7). doi:10.1001/jamanetworkopen.2020.15957

Estabrooks CA, Straus S, Flood, C., Keefe J, Armstrong P, Donner G, ... M., W. (2020). Restoring trust: COVID19 and the future of long-term care. Retrieved from https://rsc-src.ca/en/covid-19-policy-briefing/long-termcare/restoring-trust-covid-19-and-future-long-term-care

Falcão, A., Pereira, F., Pimentel, H., Teixeira, C., \& Rodrigues, V. (2017). The academic background of gerontologists and client satisfaction in long-term residential care for older persons. Educational Gerontology, 43(2), 62-72. doi:10.1080/03601277.2016.1259288

Gandal, N., Yonas, M., Feldman, M., Pauzner, A., \& Tabbach, A. D. (2020). Long-Term Care Facilities As a Risk Factor for Death Due to COVID-19. SSRN Electronic Journal. doi:10.2139/ssrn.3616760

Gardner, W., States, D., \& Bagley, N. (2020). The Coronavirus and the Risks to the Elderly in Long-Term Care. Journal of Aging \& Social Policy, 32(4-5), 310-315. doi:10.1080/08959420.2020.1750543

Harris, D. A., Archbald-Pannone, L., Kaur, J., Cattell-Gordon, D., Rheuban, K. S., Ombres, R. L., . . Mutter, J. B. Rapid Telehealth-Centered Response to COVID-19 Outbreaks in Postacute and Long-Term Care Facilities. Telemedicine and E-Health. doi:10.1089/tmj.2020.0236

He, M. Y., Li, Y. M., \& Fang, F. (2020). Is There a Link between Nursing Home Reported Quality and COVID-19 Cases? Evidence from California Skilled Nursing Facilities. Journal of the American Medical Directors Association, 21(7), 905-908. doi:10.1016/j.jamda.2020.06.016

Hsu, A. T., \& Lane, N. (2020). International Long Term Care Policy Network. Impact of COVID-19 on residents of Canada's long-term care homes - ongoing challenges and policy response. Retrieved from https://tccovid.org/

Ickert, C., Rozak, H., Masek, J., Eigner, K., \& Schaefer, S. (2020). Maintaining Resident Social Connections During COVID-19: Considerations for Long-Term Care. Gerontology and Geriatric Medicine, 6, 233372142096266. doi:10.1177/2333721420962669

Instituto Nacional de Estatística (INE, 2020). Available at: . Retrieved from http://ine.pt/. Retrieved May 20, http://ine.pt/

Kim, T. (2020). Improving Preparedness for and Response to Coronavirus Disease 19 (COVID-19) in Long-Term Care Hospitals in Korea. Infection and Chemotherapy, 52(2), 133-141. doi:10.3947/ic.2020.52.2.133

Kuzuya, M., Aita, K., Katayama, Y., Katsuya, T., Nishikawa, M., Hirahara, S., . . Japan Geriatrics Soc, S. The Japan Geriatrics Society consensus statement "recommendations for older persons to receive the best medical and long-term care during the COVID-19 outbreak-considering the timing of advance care planning implementation". Geriatr Gerontol Int. doi:10.1111/ggi.14075 
Lansbury, L. E., Brown, C. S., \& Nguyen-Van-Tam, J. S. (2017). Influenza in Iong-term care facilities. Influenza Other Respir Viruses, 11(5), 356-366. doi:10.1111/irv.12464

Lester, P. E., Holahan, T., Siskind, D., \& Healy, E. (2020). Policy Recommendations Regarding Skilled Nursing Facility Management of Coronavirus 19 (COVID-19): Lessons from New York State. Journal of the American Medical Directors Association, 21(7), 888-892. doi:10.1016/j.jamda.2020.05.058

Mas Romero, M., Avendaño Céspedes, A., Tabernero Sahuquillo, M. T., Cortés Zamora, E. B., Gómez Ballesteros, C., Sánchez-Flor Alfaro, V., . . Abizanda, P. (2020). COVID-19 outbreak in long-term care facilities from Spain. Many lessons to learn. Plos One, 15(10), e0241030. doi:10.1371/journal.pone.0241030

McMichael, T. M., Currie, D. W., Clark, S., Pogosjans, S., Kay, M., Schwartz, N. G., . . Duchin, J. S. (2020). Epidemiology of Covid-19 in a Long-Term Care Facility in King County, Washington. N Eng/ J Med, 382(21), 2005-2011. doi:10.1056/NEJMoa2005412

Mills, W. R., Buccola, J. M., Sender, S., Lichtefeld, J., Romano, N., Reynolds, K., .. Howard, S. (2020). HomeBased Primary Care Led-Outbreak Mitigation in Assisted Living Facilities in the First 100 Days of Coronavirus Disease 2019. Journal of the American Medical Directors Association, 21(7), 951-953. doi:10.1016/j.jamda.2020.06.014

Ministério do Trabalho e da Solidariedade Social. Carta Social - Rede de serviços e equipamentos 2018. In Gabinete de Estratégia e Planeamento (2019). Retrieved from http://www.gep.mtsss.gov.pt/carta-social. http://www.gep.mtsss.gov.pt/carta-social

Mitchell, S. H., Bulger, E. M., Duber, H. C., Greninger, A. L., Ong, T. D., Morris, S. C., .. Western, W. A. C.-E. P. (2020). Western Washington State COVID-19 Experience: Keys to Flattening the Curve and Effective Health System Response. J Am Coll Surg, 231(3), 316-+. doi:10.1016/j.jamcollsurg.2020.06.006

Montoya, A., Jenq, G., Mills, J. P., Beal, J., Chun, E. D., Newton, D., ... Mody, L. Partnering with Local Hospitals and Public Health to ManageCOVID-19 Outbreaks in Nursing Homes. J Am Geriatr Soc. doi:10.1111/jgs.16869

O'Caoimh, R., O'Donovan, M. R., Monahan, M. P., Dalton O'Connor, C., Buckley, C., Kilty, C., .. C Cornally, N. (2020). Psychosocial Impact of COVID-19 Nursing Home Restrictions on Visitors of Residents With Cognitive Impairment: A Cross-Sectional Study as Part of the Engaging Remotely in Care (ERiC) Project. Frontiers in Psychiatry, 11. doi:10.3389/fpsyt.2020.585373

Pereira, F., Teixeira, C., Falcão, A. R., P Rodrigues, V. M. C., \& Pimentel, H. (2019). Personal centered care in Iong term residences: how to evaluate client satisfaction?. Revista INFAD de PSicologia,, 2(2), 31-44.

Pinto, I. C., Pereira, F., \& Mateos-Campos, R. (2015). Polypharmacy and potentially inappropriate medication in elderly of Northern Portugal. Clin Ther, 37(8), 100-e101. doi:10.1016/j.clinthera.2015.05.288

PORDATA (2019). Base de Dados Portugal Contemporâneo. Available at. Retrieved from http://www.pordata.pt/.

Robb, C. E., De Jager, C. A., Ahmadi-Abhari, S., Giannakopoulou, P., Udeh-Momoh, C., McKeand, J., . . . Middleton, L. (2020). Associations of Social Isolation with Anxiety and Depression During the Early COVID19 Pandemic: A Survey of Older Adults in London, UK. Frontiers in Psychiatry, 11. doi:10.3389/fpsyt.2020.591120

Rolland, Y., Lacoste, M. H., de Mauleon, A., Ghisolfi, A., Barreto, P. D., Blain, H., \& Villars, H. (2020). Guidance for the Prevention of the COVID-19 Epidemic in Long-Term Care Facilities: A Short-Term Prospective Study. Journal of Nutrition Health \& Aging, 24(8), 812-816. doi:10.1007/s12603-020-1440-2

Santini, Z. I., Jose, P. E., York Cornwell, E., Koyanagi, A., Nielsen, L., Hinrichsen, C., . . Koushede, V. (2020). Social disconnectedness, perceived isolation, and symptoms of depression and anxiety among older Americans (NSHAP): a longitudinal mediation analysis. Lancet Public Health, 5(1), e62-e70. doi:10.1016/s2468-2667(19)30230-0

Simard, J., \& Volicer, L. (2020). Loneliness and Isolation in Long-term Care and the COVID-19 Pandemic. Journal of the American Medical Directors Association, 21(7), 966-967. doi:10.1016/j.jamda.2020.05.006 
Stall, N. M., Jones, A., Brown, K. A., Rochon, P. A., \& Costa, A. P. (2020). For-profit long-term care homes and the risk of COVID-19 outbreaks and resident deaths. Can Med Assoc J, 192(33), E946-E955. doi:10.1503/cmaj.201197

Yen, M. Y., Schwartz, J., King, C. C., Lee, C. M., Hsueh, P. R., \& Soc Taiwan Long-Term, C. (2020). Recommendations for protecting against and mitigating the COVID-19 pandemic in long-term care facilities. Journal of Microbiology Immunology and Infection, 53(3), 447-453. doi:10.1016/j.jmii.2020.04.003

Williams, J., Hadjistavropoulos, T., Ghandehari, 0. 0., Yao, X. U. E., \& Lix, L. (2015). An evaluation of a personcentred care programme for long-term care facilities. Ageing and Society, 35(3), 457-488. doi:10.1017/S0144686X13000743 